Apidologie, 1979, 10 (2), 137-148

\title{
APPROCHE BIOMÉTRIQUE DE LA VARIÉTÉ LOCALE D'APIS MELLIFICA L. DANS LE SUD TCHADIEN
}

\section{Biometrische Einordnung der lokalen Varietät von Apis mellifica L. im südlichen Tschad}

\author{
Claude GADBIN \\ Faculté des Sciences et Techniques de Saint-Jérome \\ Laboratoire de Botanique Historique et de Palynologie \\ Rue Henri-Poincaré - 13397 Marseille Cedex 4 \\ J.-M. CORNUET, J. FRESNAYE \\ Station Expérimentale d'Apiculture, I.N.R.A. \\ F. 84140 Montfavet

\section{SUMMARY} \\ BIOMETRICAL STUDY OF THE LOCAL VARIETY \\ OF APIS MELLIFICA IN SOUTHERN CHAD
}

\begin{abstract}
Biometrical studies and statistical analysis of honeybee samples from Chad allow us to conclude that honeybees of this area belong to the race Apis mellifica adansonii. It was not possible to prove the existence of differenciated populations inside the Chad honeybee, but it appears that Apis mellifica adansonii is perfectly marked off from the honeybee races situated in more northern areas, as well as from european honeybee races by means of the discriminant factor analysis.
\end{abstract}

\section{RÉSUMÉ}

Les études biométriques et les analyses statistiques d'échantillons d'abeilles du Tchad méridional nous permettent de conclure à l'appartenance des abeilles de cette région à la race Apis mellifica adansonii. S'il n'a pas été possible de mettre en évidence l'existence de populations différenciées au sein de l'Abeille du Tchad, il apparait par contre qu'Apis mellifica adansonii se distingue parfaitement des races d'abeilles situées dans les régions plus septentrionales, ainsi que des races d'abeilles d'Europe au moyen de l'analyse factorielle discriminante. 


\section{INTRODUCTION}

L'abeille africaine est connue et utilisée par l'homme depuis la préhistoire. Cependant, et contrairement aux races européennes dont les caractéristiques et la répartition géographique sont connues avec précision, l'étude de la morphologie des races d'Afrique et des régions qu'elles occupent est à peine ébauchée. LATREILLE (1804) n'a décrit que le seul type $A . m$. adansonii pour toute l'Afrique centrale. KerR et Portugal-Araujo (1958) et SMith (1961) ont défini cette race avec des bandes jaunes sur les trois premiers segments abdominaux, un scutellum jaune et des poils jaunes sur l'ensemble du corps, mais constatent l'extrême variabilité de la couleur. SMITH décrit en outre des races distinctes de ce type, A. m. monticola abeille de couleur foncée des régions montagneuses de l'Afrique orientale; ainsi que $A . m$. litorea petite abeille à bandes jaunes qui vit sur la côte de Tanzanie. A.m. scutellata est une abeille jaune, établie dans les régions situées entre l'Afrique centrale et l'Afrique du sud.

RUTTNER (1975) a obtenu une très bonne différenciation de ces races en utilisant une analyse discriminante à partir de 33 caractères morphologiques, démontrant ainsi le bien-fondé de la diversité des dénominations. FLETCHER (1978) a recensé les connaissances concernant la biologie et le comportement de ces races.

Nous pouvons constater avec RUTTNER (1976) que l'on ignore encore tout des limites exactes des régions occupées par les différentes races d'abeilles en Afrique, et particulièrement en Afrique centrale. La présente étude biométrique a été réalisée avec des abeilles du Tchad méridional afin de combler partiellement les lacunes concernant leurs caractères morphologiques, de les situer au sein de l'espèce et de connaitre les possibilités de leur appliquer nos méthodes habituelles pour la discrimination des races d'abeilles.

\section{MATÉRIEL ET MÉTHODES}

\section{A. Origine géographique des échantillons étudiés}

Les abeilles ont été récoltées dans une région couvrant une superficie de plus de $1000 \mathrm{~km}^{2}$, située aux alentours de la capitale, N'DJAMENA. Les villages de Linia, Drogana, Mailao, Rokum, sont situés au bord des fleuves délimitant cette zone, le Logone, le Chari et le Bahr Linia (fig. 1). Cette région est uniformément plate, couverte d'une végétation de type savane arbustive, localement modifiée en steppe à mimosées surtout d'origine anthropique, et en forêt claire au voisinage des fleuves.

\section{B. Méthodes de mesures et caractères biométriques}

Les analyses biométriques ont porté sur les cinq caractères les plus significatifs pour la discrimination des races d'abeilles en Europe (GOETZE 1963, RUTTNER 1968, 1973) et que nous utilisons également depuis de nombreuses années (Fresnaye 1965, Tomassone et Fresnaye 1971, Cornuet et al. 1975). Les mesures sont effectuées sous microscope stéréoscopique selon les méthodes décrites par ailleurs en détail (FRESNAYE 1974).

Elles concernent :

1) la coloration. Il s'agit de la largeur des taches ou de la bande jaune sur le $2^{e}$ tergite abdominal. Elle peut couvrir de 0 à $2,4 \mathrm{~mm}$. Elle est mesurée au grossissement $\times 12$; 
2) la longueur des poils (" pilosité ") sur le $5^{\mathrm{e}}$ tergite abdominal, mesurée au grossissement $\times 50$; $\times 12$;

3) la largeur du tomentum sur le $4^{\mathrm{e}}$ tergite abdominal; mesure de la bande pileuse au grossissement

4) la longueur de la langue. La glosse est mise en extension à l'aide de pinces à dissection et les mesures effectuées au grossissement $\times 12$;

5 et 6) les mesures $A$ et $B$ qui composent l'index cubital sont utilisées individuellement afin d'éviter toute perte d'information. Elles sont effectuées au grossissement $\times 50$ à l'aide du dispositif décrit par RUTTNER (1963).

\section{Mesure de la taille des cellules dans les rayons naturels}

Les mesures de la taille des cellules ont été réalisées sur des groupes de 10 cellules voisines à l'aide d'un oculaire micrométrique sous le microscope stéréoscopique. Quatre séries de 10 mesures ont été exécutées, les résultats obtenus sur 10 cellules rapportés à l'unité, puis le nombre de cellules au $\mathrm{dm}^{2}$ calculé pour les 2 faces du rayon en assimilant la surface cellulaire à un hexagone régulier.

\section{Méthodes statistiques}

L'unité d'observation est la colonie d'abeilles, sur un échantillon de laquelle sont mesurée les 6 variables. Les variables : coloration, pilosité, tomentum, longueur de la langue, sont mesurées sur 10 abeilles. Les variables $\mathrm{A}$ et $\mathrm{B}$ de l'index cubital sont mesurées sur 30 abeilles.

Outre les statistiques élémentaires, deux types d'analyses statistiques ont été effectuées à l'aide de l'ordinateur :

- analyse en composantes principales pour détecter une éventuelle structure au sein des colonies tchadiennes;

- analyse factorielle discriminante pour situer l'abeille tchadienne au sein de l'espẻce.

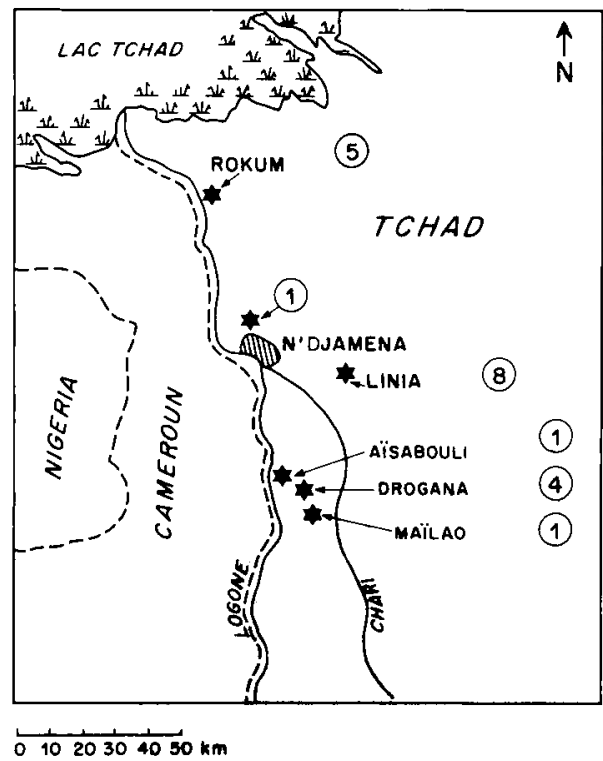

Fig. 1. - Plan de la région du Tchad où les prélèvements ont été effectués.

Étoiles : sites de prélèvement des échantillons.

Chiffres dans un cercle : nombre d'échantillons par site.

Aвв. 1. - Karte der Region des Tschad, wo die Proben entnommen wurden.

Sterne : Orte der Probenentnahme.

Ziffern im Kreis : Anzahl der Proben je Standort. 


\section{RÉSULTATS}

\section{1) Taille des cellules dans les rayons naturels}

La taille relative des abeilles des différentes races peut être exprimée par la dimension des cellules ou le nombre des cellules par $\mathrm{dm}^{2}$ dans les rayons naturels. D'après nos mesures l'abeille du Tchad méridional construit en moyenne 1045 cellules d'ouvrières au $\mathrm{dm}^{2}$ sur les 2 faces. Comparativement aux abeilles d'Europe mentionnées dans le tableau I, cette abeille est donc de petite taille. Cette taille est comparable à celle attribuée à $A$. m. adansonii par d'autres auteurs.

\section{2) Caractéristiques biométriques des abeilles du Tchad}

\section{a) Caractères morphologiques}

Les valeurs moyennes des caractères morphologiques sont indiquées dans le tableau II qui présente également à titre de comparaison les moyennes des caractères pour les races étudiées à la station d'Apiculture de Montfavet. (TOMAssone et FresNAYE 1971; CoRNUET et al. 1975.) Avec une bande jaune dont la largeur moyenne est de 1,32 mm, l'abeille du Tchad est à classer parmi les races d'abeilles de couleur jaune.

TABL. 1. - Dimensions alvéolaires et nombre au $\mathrm{dm}^{2}$ chez quelques races d'Apis mellifica.

ТАв. 1. - Zelldurchmesser und Zellzahl pro $\mathrm{dm}^{2}$ bei einigen

Rassen von Apis mellifica.

\begin{tabular}{|c|c|c|c|c|}
\hline \multirow[b]{2}{*}{$\begin{array}{l}\text { Race } \\
\text { Rasse }\end{array}$} & \multirow{2}{*}{$\begin{array}{l}\text { Origine } \\
\text { Herkunft }\end{array}$} & \multirow{2}{*}{$\begin{array}{l}\text { Auteur } \\
\text { Autor }\end{array}$} & \multicolumn{2}{|c|}{ + } \\
\hline & & & $\begin{array}{c}\text { Nombre } \\
\text { Zahl }\end{array}$ & $\begin{array}{l}\text { Dimension } \\
\text { Durchmesser }\end{array}$ \\
\hline adansonii .. & Tchad & GADBIN & 1045 & 4,7 \\
\hline adansonii .. & $\begin{array}{l}\text { Afrique occidentale et centrale } \\
\text { West-und Zentralafrika }\end{array}$ & FRESNAYE & 1050 & 4.68 \\
\hline adansonii .. & Angola & Portugal araujo & 1008 & 4,8 \\
\hline adansonii .. & Tanganyka & SMITH & 1000 & 4,8 \\
\hline ligustica ... & $\begin{array}{l}\text { Centre Italie } \\
\text { Italien (Zentrum) }\end{array}$ & Alber & 830 & 5,27 \\
\hline mellifica ... & $\begin{array}{l}\text { Nord-ouest Italie } \\
\text { Nordwesitalien }\end{array}$ & Alber & 800 & 5,37 \\
\hline carnica .... & $\begin{array}{l}\text { Nord-est Italie } \\
\text { Nordostitalien }\end{array}$ & AlBer & 760 & 5,51 \\
\hline
\end{tabular}


La pilosité est très courte : $0,17 \mathrm{~mm}$. Le tomentum est plutôt large mais sa faible densité le rend difficile à mesurer et il sera sans doute nécessaire de réviser les conceptions de prise des mesures de ce caractère. La langue est très courte : 5,45 mm. L'index cubital est élevé : 2,35 . Les éléments $\mathrm{A}(0,489 \mathrm{~mm})$ et $\mathbf{B}(0,208 \mathrm{~mm})$ de l'index cubital sont les plus faibles parmi les races que nous ayons étudiées et constituant une nouvelle indication de la petite taille de cette race.

\section{b) Recherche de populations différenciées chez l'Abeille du Tchad}

Lors des études précédentes déjà mentionnées nous avons pu mettre en évidence l'existence de populations différenciées au sein de la race $A . m$. mellifica. Nous avons recherché si de telles populations pouvaient être détectées au sein des colonies d'abeilles du Tchad en les soumettant à une analyse en composantes principales. Aucun groupement remarquable ni aucune subdivision n'ont été révélés par l'analyse.

\section{c) Discrimination de l'Abeille du Tchad au sein de l'espèce Apis mellifica}

Afin de déterminer les possibilités de différencier l'Abeille du Tchad d'autres races d'abeilles d'Afrique, d'Europe ou d'Asie nous avons utilisé l'analyse discriminante, dont nous avions déjà pu apprécier la grande efficacité lors de nos études précédentes. Les abeilles du Tchad sont comparées à des groupes d'abeilles des différentes races pour lesquelles le fichier de la Station Expérimentale d'A piculture de l'I.N.R.A. possède des références biométriques valables.

L'analyse discriminante à 6 caractères biométriques que nous avons effectuée permet une discrimination très nette de l'abeille du Tchad parmi les autres races représentées. Les trois premiers axes discriminants absorbent $97 \%$ de la variabilité totale. Sur le plan factoriel Z 1-Z 2 (fig. 2) les échantillons d'abeilles du Tchad sont bien groupés en bas et à gauche. Très proche, mais cependant séparés, vient ensuite $A$. m. lamarkii; puis $A$. m. sahariensis, $A . m$. syriaca et $A$. m. ligustica, qui constituent le groupe des races de couleur jaune. Les races de couleur foncée sont groupées dans le haut du plan de projection. Le plan factoriel Z 1-Z 3 (fig. 3) présente également un très bon groupement des abeilles du Tchad ainsi qu'une bonne discrimination vis-à-vis des autres races étudiées, sauf pour A. m. lamarkii qui est englobée dans le groupe tchadien.

Le caractère " couleur " est considéré par divers spécialistes comme secondaire dans la différenciation raciale des abeilles. Il est déterminant dans la dichotomie des races de l'abeille, aussi bien visuellement que dans les analyses statistiques. Il est donc très utile d'effectuer des analyses discriminantes dans lesquelles on a supprimé le caractère "couleur ". Dans cette analyse à 5 caractères biométriques, les colonies sont regroupées en "souches" représentant soit une race, soit une population locale. Les trois premiers axes discriminants absorbent $98 \%$ de la variabilité totale. Sur le plan factoriel Z 1-Z 2 (fig. 4) les échantillons d'abeilles du Tchad sont situés en haut et à droite. Bien séparée, $A$. m. lamarkii vient ensuite, puis $A . m$. sahariensis, $A . m$. intermissa, $A$. m. syriaca légèrement à gauche, $A$. m. ligustica avec $A$. m. carnica, au bas à gauche $A . m$. caucasica et à droite $A, m$. mellifica. On peut remarquer que la distribu- 


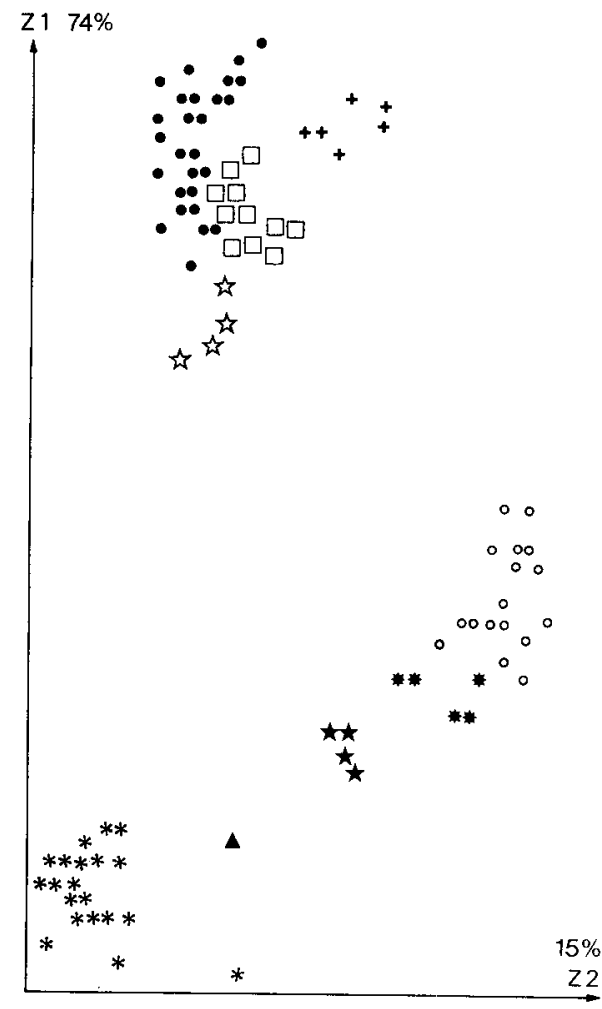

FIG. 2. - Analyse discriminante à 6 caractères biométriques. Projection des colonies sur les plans factoriels Z 1-Z 2 .

AвB. 2. - Diskriminanzanalyse mit 6 biometrischen Merkmalen. Projektion der Völker auf die Faktorenebenen $Z 1-Z 2$.

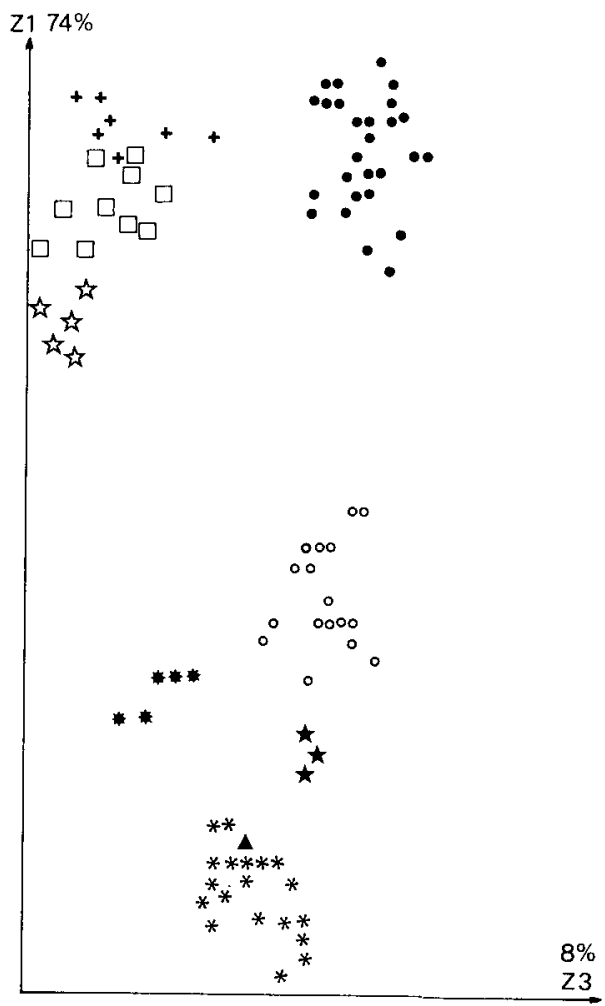

FIG. 3. - Analyse discriminante à 6 caractères biométriques. Projection des colonies sur les plans factoriels $Z 1-Z 3$.

Aвв. 3. - Diskriminanzanalyse mit 6 biometrischen Merkmalen. Projektion der Völker auf die Faktorenebenen Z 1-Z3.
* Apis mellifica adansonii

Apis mellifica carnica

+ Apis mellifica caucasica

放 Apis mellifica intermissa

A Apis mellifica lamarkii
- Apis mellifica ligustica

- Apis mellifica mellifica

$\star$ Apis mellifica sahariensis

* Apis mellifica syriaca 


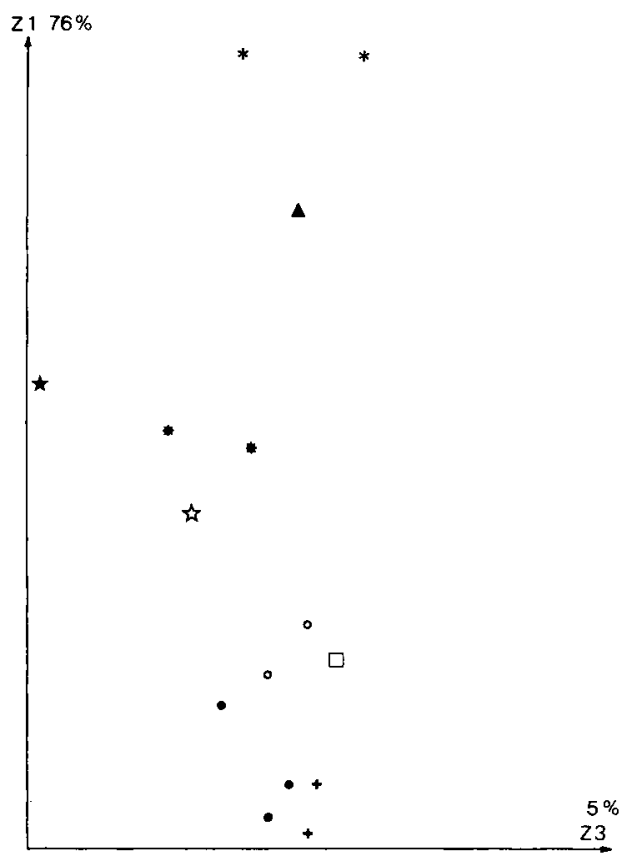

FIG. 4. - Analyse discriminante

à 5 caractères biométriques

(sans la "couleur ").

Projection des souches

sur les plans factoriels Z 1-Z 2 .

(Légende figure 2).

Авв. 4. - Diskriminanzanalyse mit 5 biometrischen Merkmalen (ohne a Farbe w.

Projektion der Stämme

auf die Faktorenebenen Z I-Z 2.

(Legende s. Abb. 2).

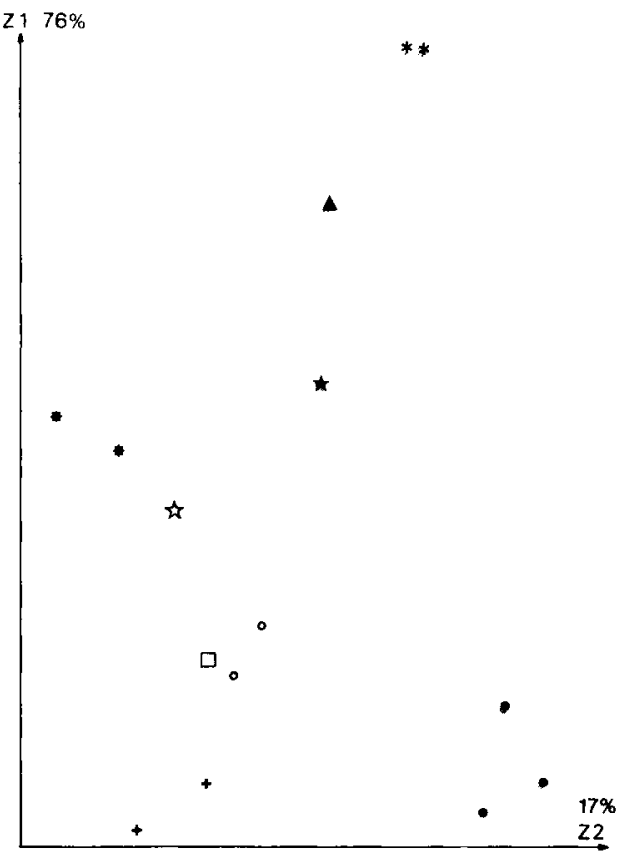

FIG. 5. - Analyse discriminante

à 5 caractères biométriques (sans la " couleur "). Projection des souches sur les plans factoriels $Z 1-Z 3$. (Légende figure 2).

Aвв. 5. - Diskriminanzanalyse mit 5 biometrischen Merkmalen (ohne " Farbe ").

Projektion der Stämme

auf die Faktorenebenen Z 1-Z 3. (Legende s. Abb. 2).

tion des races selon l'axe $\mathrm{Z} 1$ reflète assez bien le gradient de la latitude de leur situation géographique : les races africaines tropicales et subtropicales sont opposées aux races européennes.

\section{DISCUSSION}

Les analyses biométriques et les analyses statistiques que nous avons effectuées sur des échantillons d'abeilles du Tchad méridional permettent de définir la position de 
cette race comparativement aux autres races au sein d'Apis mellifica du point de vue morphologique.

L'abeille du Tchad est petite; cela est démontré par la taille des cellules dans les rayons : 1045 cellules au $\mathrm{dm}^{2}$ pour un diamètre de $4,7 \mathrm{~mm}$ alors que l'on trouve pour les abeilles d'Europe 830 cellules au dm $\mathrm{dm}^{2}$ et $5,27 \mathrm{~mm}$ de diamètre pour $A$. m. ligustica; 800 cellules au dm ${ }^{2}$ et $5,37 \mathrm{~mm}$ de diamètre pour $A$. m. mellifica; 760 cellules au $\mathrm{dm}^{2}$ et $5,51 \mathrm{~mm}$ de diamètre pour A. m. carnica (Alber 1956). Plusieurs caractères biométriques de notre étude confirment sa petite taille. La langue de cette abeille est très courte : $5,45 \mathrm{~mm}$. Sмітн (1961) a trouvé une langue de $5,85 \mathrm{~mm}$ pour des abeilles de régions voisines. Au niveau de l'abeille cette marge est importante, mais il faut remarquer que des différences méthodologiques peuvent entrainer une variation notable des mesures. Cependant la différence de longueur de la langue entre $A . m$. adansonii et $A . m$. mellifi$c a$ est encore plus importante. Rappelons qu'A. m. mellifica est l'abeille européenne dont la langue est la plus courte, $6,28 \mathrm{~mm}$ en moyenne dans nos études.

L'abeille du Tchad est de couleur jaune (moyenne de nos mesures $1,32 \mathrm{~mm}$ ); elle est relativement peu variable si l'on considère les appréciations des différents auteurs concernant les abeilles d'Afrique centrale. Il semble que l'Abeille du Tchad soit peu soumise à l'influence des races d'abeilles plus foncées situées dans les montagnes plus à l'est et au sud.

La pilosité est très courte, en moyenne $0,17 \mathrm{~mm}$. Elle est voisine de celle de $A . m$. lamarkii, A. m. sahariensis et A. $m$. syriaca de nos études antérieures ou des autres races de l'Afrique tropicale étudiées par RUTTNER (1975). Elle est beaucoup plus courte que celle des races européennes et notamment $A$. $m$. mellifica dont la moyenne est de $0,46 \mathrm{~mm}$.

Le tomentum est assez large, mais nous avons déjà signalé sa très faible densité ainsi que la nécessité d'envisager de modifier la méthode de mesure de ce caractère. En effet, la méthode actuellement utilisée a été étudiée pour les races européennes dont le tomentum est nettement marqué.

L'index cubital moyen est de 2,348. Il est proche de celui des races d'Afrique centrale étudiées par RuTTNER. Les mesures A et B de l'index cubital sont les plus courtes parmi les races que nous ayons étudiées.

L'analyse en composantes principales que nous avons effectuée dans le but de détecter des populations différenciées morphologiquement au sein de l'Abeille du Tchad n'a pas abouti à un résultat positif. Ceci peut paraître surprenant si l'on compare avec les résultats trouvés en France. Des groupes de colonies distantes d'environ $100 \mathrm{~km}$ sont parfaitement discriminés sur la base des mêmes caractères morphologiques (CORNUET et al. 1978) et il apparaît même un isolement génétique entre 2 ruchers distants de $8 \mathrm{~km}$ en région montagneuse. 
Différentes hypothèses peuvent être avancées pour expliquer cette nondiscrimination de l'Abeille tchadienne :

- les caractères biométriques choisis en raison de leur efficacité dans la distinction des races européennes ne possèdent pas le même pouvoir discriminant chez les abeilles africaines (RUTTNER 1975, RUTTNER et al. 1978).

- le comportement de la race adansonii est très différent de celui des races européennes. En particulier, il faut mentionner cet essaimage très spécial au cours duquel de nombreux petits essaims se regroupent pour former des masses énormes avec de nombreux individus sexués (mâles et reines) puis se subdivisent à nouveau avant de nidifier (CHANDLER 1976). Il suffit de rappeler la vitesse avec laquelle les abeilles " africanisées " se sont répandues en Amérique du Sud (environ $200 \mathrm{~km}$ par an) pour comprendre que le brassage génétique peut se faire à une échelle bien plus vaste en Afrique centrale qu'en Europe. De plus, il n'existe pas d'obstacle naturel susceptible d'entraver les échanges entre les diverses colonies constituant notre échantillonnage.

Ce brassage génétique plus intense serait peut-être la cause de la variabilité des caractères morphologiques au sein d'une même colonie déjà signalée par SMITH (1961) et que nous avons retrouvée en particulier dans les histogrammes de l'index cubital.

Il faut enfin souligner que la méthode d'analyse utilisée ici est légèrement différente de celle employée par Cornuer et al. (1978) en ce qui concerne le niveau de regroupement des observations (les individus au sens statistique étaient alors les abeilles et non les colonies) et, pour cette raison, est, a priori, moins performante.

La proximité dans nos analyses discriminantes de l'Abeille du Tchad et d'A. $m$. lamarkii est remarquable. Elle a déjà été constatée par SMITH (1972) qui considère cette dernière comme un écotype d'A. $m$. adansonii, $A . m$. sahariensis n'est guère plus éloignée sur le graphique et RuTTNER et al. (1978) la considèrent également commè très voisine d' $A$. $m$. adansonii et comme un vestige de l'époque où le Sahara était encore une région de savanes reliées à l'Afrique centrale. La différence morphologique actuelle avec $A . m$. adansonii pourrait être due à une dérive génétique causée par l'isolement.

Il est possible de donner une interprétation au fait que le facteur 1 de l'analyse sans la couleur reflète la position latitudinale des races étudiées. En effet, le premier facteur dans ce type d'analyse est très souvent un facteur de taille (LEBART et FENELON 1973). Or, les abeilles sont de plus en plus grandes au fur et à mesure que l'on s'éloigne de l'équateur (tableau II) suivant en cela une loi générale de l'évolution qui veut que la taille augmente avec la latitude. 


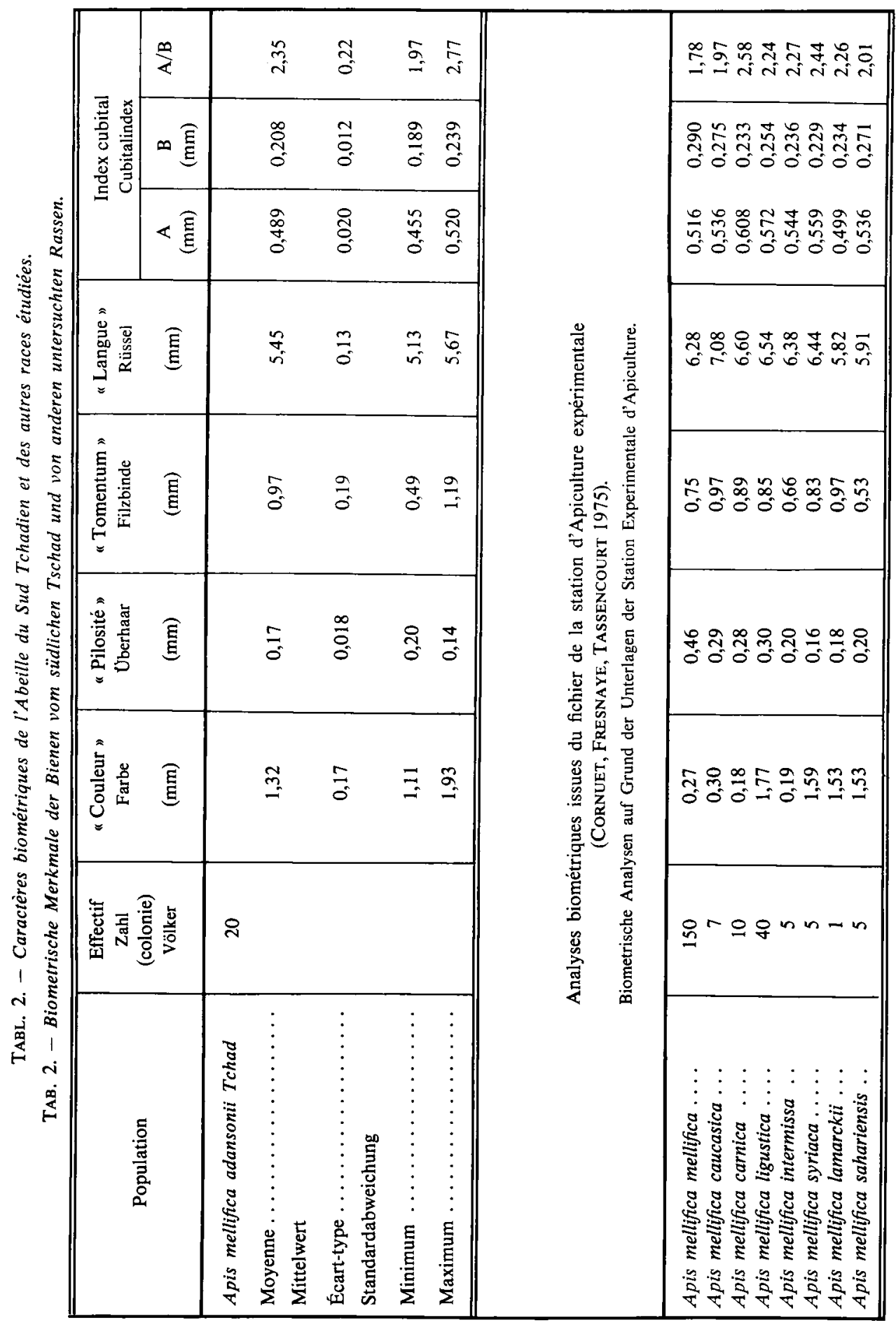




\section{ZUSAMMENFASSUNG}

Die biometrischen Studien und statistischen Analysen, die wir bei Bienenproben vom südlichen Tschad durchgeführt haben, erlauben uns, auf die Zugehörigkeit der Bienen dieser Region zur Rasse Apis mellifica adansonii zu schliessen.

Die Mittelwerte der untersuchten biometrischen Merkmale sind foigende : Farbe 1,32 mm (was einer Biene von gelber Farbe entspricht), Behaarung $0,17 \mathrm{~mm}$, Tomentbinde $0,97 \mathrm{~mm}$ (aber ihre sehr geringe Dichte macht die Messung schwierig, so dass eine Modifikation der Messmethode für diese Rasse nötig sein wird), der Rüssel ist mit $5,45 \mathrm{~mm}$ gleichmässig sehr kurz, der Cubitalindex beträgt 2,35, die Aderstükke $\mathrm{A}$ und $\mathrm{B}$ sind kurz. Die Biene ist klein.

Eine an Tschad-Völkern durchgeführte Analyse nach " principal components " hat keine besondere Differenzierung ergeben, trotz der Entfernung, welche die betrachteten Zonen trennen. Die Erklärung dafür kann eine ungenügende Effizienz der biometrischen Merkmale sein, welche zunächst für die Unterscheidung der europäischen Rassen gewählt wurden, oder wohl auch eine relative Gleichförmigkeit der Völker, die sich aus einer intensiveren genetischen Vermischung infolge des starken Wandertriebes der Andansonii-Rasse ergeben hat.

Die angewendete Methode der Diskriminanzanalyse erlaubt, Apis mellifica adansonii vollkommen von den Bienenrassen der nördlichen Gegenden Afrikas ebenso wie von den Bienenrassen Europas zu unterscheiden. Die Nähe der Rassen Apis mellifica lamarkii und Apis mellifica sahariensis in den Faktorentabellen der Diskriminanzanalyse erlaubt, diese Rassen als mit der Apis mellifica adansonii sehr nahe verwandt anzusehen, wie schon RUTTNER und SMITH gezeigt haben.

\section{BIBLIOGRAPHIE}

Alber M. A., 1956. - La misura della celle caracteristica razziale. L'Apicoltore d'Italia, 23, 193-196.

Chandere M. T., 1976. - The african honeybee. Apis mellifera adansonii, the biological basis of its management. In : Apiculture in tropical climates, Londres, I.B.R.A., 1976, 61-68.

Cornuet J.-M., Fresnaye J., Tassencourt L., 1975. - Discrimination et classification de populations d'abeilles à partir de caractères biométriques. Apidologie, 6 (2), 145-187.

Cornuet J.-M., Fresnaye J., Lavie P., 1978. - Étude biométrique de 2 populations d'abeilles cévenoles. Apidologie, 9 (1), 41-55.

Fletcher D. J. C., 1978. - The African Bee, Apis mellifera adansonii, in Africa, Annu. Rev. Entomol., 23, 151-171.

FRESNAYE J., 1965. - Étude biométrique de quelques caractères morphologiques de l'abeille noire française (Apis mellifica mellifica), Ann. Abeille, 8 (4), 271-283.

Fresnaye J., 1974. - Biométrie de l'Abeille. Bull. techn. apic. OPIDA, 3 : I-42.

Goetze G., 1963. - Die Honigbiene in natürlicher und künstlicher Zuchtauslese Paul PAREy, Hamburg$212 \mathrm{p}$.

Kerr W. E., Portugal Araujo V. de, 1958. - Racas de abelhas de Africa. Garcia de Orta, 6 (1), 53-59.

LATREILlE P.-A., 1804. - Notice des espèces d'abeilles vivant en grandes sociétés ou abeilles proprement dites et descriptions d'espèces nouvelles. Ann. Mus. Hist. Nat., Paris, 5, 161-178.

Lebart L., Fenelon J.-P., 1973. - Statistique et informatique appliquées. Dunod Paris.

Portugal de Araujo V. de P., 1956. - Notas bionomicas sobre Apis mellifera adansonii Latr. Dusenia. 7 (2), 91-102.

RUTTNER F., 1973. - Zuchttechnik und Zuchtauslese bei der Biene. Ehrenwirth Verlag, München, 138 p.

RutTner F., 1968. - Les races d'abeilles. In : Traité de biologie de l'Abeille, R. Chauvin ed. Paris, Masson, $\mathrm{t} 1: 27-44$. 
RUtTNer F., 1975. - Les races d'abeilles de l'Afrique. Congr. Int. Apiculture XXV. Grenoble, 1975 : 347 367.

RUTTNER F., 1976. - Honeybees of the tropics : their variety and characteristics of importance for apiculture. Apiculture in tropical climates. Ed. E. Crane, London, B.R.A., 1976:41-46.

RutTNer F., TAssencourt L. et LouveauX J., 1978. - Biometrical statistical analysis of the geographic variability of Apis mellifera L., Apidologie, 9 (4), 363-381.

SMrth F. G., 1961. - The races of honey bee in Africa. Bee Wo, $t$, 42 (10) : 255-260.

Smith F. G., 1972. - Les races africaines d'Apis mellifica. Bull. Sci. Apimondia 363-366.

TOMAssone R., Fresnaye J., 1971. - Étude d'une méthode biométrique et statistique permettant la discrimination et la classification de populations d'abeilles (Apis mellifica L.) Apidologie, 2 (1), 49-65. 